\title{
SOROPREVALÊNCIA DE LEPTOSPIROSE EM FÊMEAS BOVINAS EM IDADE REPRODUTIVA NO ESTADO DA BAHIA
}

\author{
F.C.S. Oliveira' ${ }^{1}$, S.S. Azevedo ${ }^{2}$, S.R. Pinheiro ${ }^{1}$, S.A.R.A. Viegas ${ }^{3}$, C.S.A. Batista ${ }^{1}$, C.P. Coelho ${ }^{1}$, \\ Z.M. Moraes ${ }^{1}$, G.O. Souza ${ }^{1}$, A.P. Gonçales ${ }^{1}$, C.A.S. Almeida ${ }^{1}$, S.A. Vasconcellos ${ }^{1}$
}

${ }^{1}$ Universidade de São Paulo, Faculdade de Medicina Veterinária e Zootecnia, Departamento de Medicina Veterinária Preventiva e Saúde Animal, Laboratório de Zoonoses Bacterianas, Av. Prof. Dr. Orlando Marques de Paiva, 87, CEP 05508-270, São Paulo, SP, Brasil. E-mail: faucarol@gmail.com

\section{RESUMO}

\begin{abstract}
O objetivo do presente trabalho foi determinar a soroprevalência da leptospirose em fêmeas bovinas em idade reprodutiva no Estado da Bahia. A amostragem foi delineada para a determinação da prevalência de propriedades positivas (focos) e de animais soropositivos para a leptospirose. O Estado foi dividido em quatro regiões ou estratos amostrais, nos quais foram examinadas 10.823 fêmeas bovinas com idade $\geq 24$ meses distribuídas em 1.414 propriedades. A reação de Soroaglutinação Microscópica (SAM), empregando 23 sorovares de Leptospira spp. como antígenos, foi utilizada como teste diagnóstico. O rebanho foi considerado foco quando apresentou pelo menos um animal soropositivo. As prevalências de foco e de animais soropositivos no Estado foram de 77,93\% [IC 95\% = 75,73\% - 79.99\%] e 45,42\% [IC 95\% = 42,00\% - 48,88\%], respectivamente. O sorovar mais frequente foi o Hardjo (Hardjoprajitno), em 34,49\% [IC 95\% = 31,93\% - 37,14\%] das propriedades positivas e 14,95\% [IC 95\% = 12,59\% - 17,67\%] de animais soropositivos de diferentes regiões.
\end{abstract}

PALAVRAS-CHAVE: Leptospira spp., bovinos, prevalência, epidemiologia (controle).

\section{ABSTRACT}

SEROPREVALENCE OF LEPTOSPIROSIS IN REPRODUCTIVE-AGE BOVINE FEMALES IN THE STATE OF BAHIA, NORTHEASTERN BRAZIL. The aim of this work was to determine the seroprevalence of leptospirosis in reproductive-age bovine females in Bahia State, Northeastern Brazil. The sampling was delineated for the determination of the prevalence of seropositive animals as well as herds positive for bovine leptospiroses (foci). The state was divided into 4 regions or sampling strata in which 10,823 bovine females aged $\geq 24$ months allocated in 1,414 herds were sampled. The microscopic agglutination test (MAT), using 23 Leptospira spp. serovars as antigens, was employed as a diagnostic test. The herd was considered positive if at least one animal was seropositive. The prevalences of positive herds and seropositive animals in the state were $77.93 \%$ [75.73\%-79.99\%] and 45.42\% [42.00\%-48.88\%], respectively.Serovar Hardjo (Hardjoprajitno) was the most frequent, with $34.49 \%$ [31.93\%-37.14\%] of positive herds and $14.95 \%$ [ $12.59 \%-17.67 \%$ ] of seropositive animals in the different regions.

KEY WORDS: Leptospira spp., bovine, prevalence, epidemiology (control).

\section{INTRODUÇÃO}

A leptospirose é uma zoonose de curso agudo a crônico que afeta diversas espécies de animais domésticos, silvestres e os seres humanos, que assume considerável importância em todo o mundo tanto como problemas econômicos como de saúde pública (FAINE et al., 1999).
A leptospirose apresenta distribuição geográfica cosmopolita, porém a sua ocorrência é maior em países de clima tropical e subtropical nos quais favorecem a sobrevivência das leptospiras. Apresenta-se usualmente na forma endêmica com morbidade alta em todos os países em que tem sido estudada. Os sorovares envolvidos podem variar de região para região dependendo das espécies animais de contato,

${ }^{2}$ Universidade Federal de Campina Grande, Unidade Acadêmica de Medicina Veterinária, Patos, PB, Brasil.

${ }^{3}$ Universidade Federal da Bahia, Faculdade de Medicina Veterinária, Salvador, BA, Brasil. 
dos sorovares existentes, das condições ambientais e climáticas, além do manejo e das oportunidades de infecção direta ou indireta (ELLIS, 1984). A manutenção de leptospiras na natureza é assegurada pelos animais portadores domésticos e silvestres (FAINE et al., 1999).

Osorovar de Leptospira spp. mais frequentemente encontradoem bovinoséoHardjo, do qual os bovinos são hospedeiros primários de manutenção. Dois tipos do sorovar Hardjo sorologicamente idênticos, mas geneticamente distintos, são aceitos: Leptospira interrogans sorovar Hardjo tipo Hardjoprajitno e Leptospira borgpetersenii sorovar Hardjo tipo Hardjobovis. O sorovar Hardjo tipo Hardjobovis é comum em populações de bovinos de muitas regiões do mundo. O tipo Hardjoprajitno foi isolado primeiramente em bovinos no Reino Unido (GROOMs, 2006).

Os sinais clínicos da leptospirose em bovinos são muito variados, incluindo febre, diarreia, anemia, icterícia e hemoglobinúria. As infecções agudas resultam em infertilidade, abortamentos, natimortalidade, nascimento de bezerros fracos e mastite (ELLIS, 1994). ElLIS et al. (1985) relataram que vacas infectadas podem requerer até seis coberturas para conceber. Abortos subsequentes podem ocorrer devido a persistência do agente no trato genital (ELLIS et al.,1985). Em vacas com aptidão leiteira, podehaver a infecção da glândula mamária e o quadro clínico é o de mastite atípica, com sensível diminuição da secreção láctea em até $80 \%$ ou mais do volume, retornando a produção normal em 10 a 15 dias, úbere flácido e leite manchado por coágulos de sangue (Fundação NaCiOnAL dE SAÚde, 1995).

O controle da leptospirose animal consiste em integração de medidas profiláticas instituídas simultaneamente nos três níveis da cadeia de transmissão: fontes de infecção (vertebrados infectados), vias de transmissão (água, solo e fômites contaminados) e susceptíveis (vertebrados não infectados enãoimunizados) (FAINE et al., 1999).

Considerando-se que aproximadamente $40 \%$ da população bovina no Nordeste (11.385.723 animais) (IBGE, 2007) está no Estado da Bahia, é importante o conhecimento da ocorrência e distribuição espacial das doenças transmissíveis que acometem a eficiência reprodutiva dos rebanhos bovinos do Estado, dentre as quais se destaca a leptospirose. De fato, com a obtenção da informação das variantes sorológicas presentes e da sua distribuição espacial, será possível o direcionamento de um programa de controle regionalizado e dirigido para as respectivas sorovariedades predominantes. Deste modo, o presente trabalho foi delineado com o objetivo de determinar a soroprevalência de leptospirose em fêmeas bovinas em idade reprodutiva no Estado da Bahia.

\section{MATERIALEMÉTODOS}

\section{População estudada}

Foi utilizado obanco de soro oriundo do estudo da situação epidemiológica da brucelose bovina no Estado da Bahia, realizado como parte do Programa Nacional de Controle e Erradicação da Brucelose e Tuberculose (Alves, 2007) e planejado pelo Ministérioda Agricultura, Pecuária e Abastecimento(MAPA), em colaboraçãocoma Agência de Defesa Agropecuária do Estado da Bahia (ADAB). Nesse estudo foram analisados 10.823 soros de fêmeas bovinas com idade igual ou superior a 24 meses, provenientes de 1.414 propriedades, sorteadas dentro de quatro estratos amostrais (Fig. 1) doEstado. Em propriedades com até 99 fêmeas com idade igual ou superior a 24 meses foram amostradas 10 delas e naquelas com $100 \mathrm{ou}$ mais foram amostradas 15 delas.

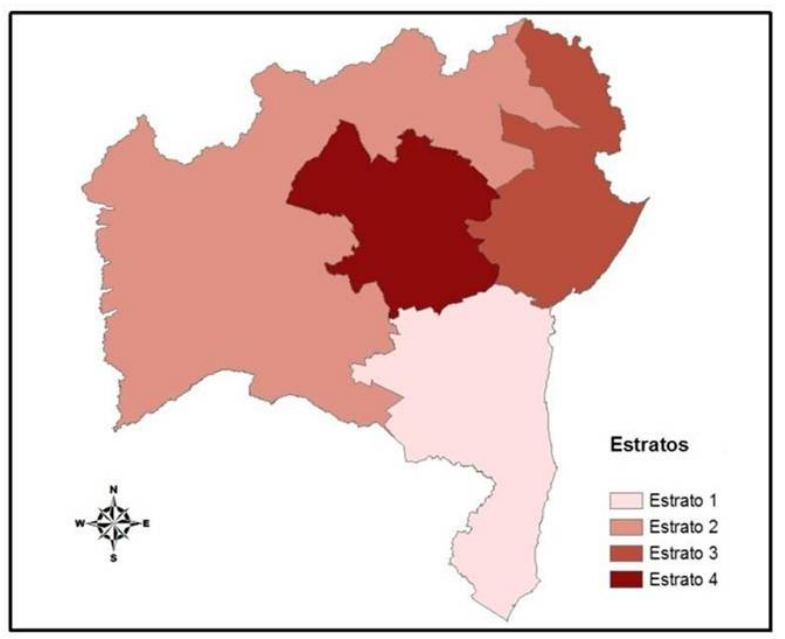

Fig.1 - Mapa do Estado da Bahia com a subdivisão em quatro estratos amostrais.

\section{Diagnóstico sorológico da leptospirose}

O diagnóstico da leptospirose foi realizado pela técnica de soroaglutinação microscópica SAM, de acordo com GALTONetal.(1965) eCole etal.(1973), com uma coleção de antígenos vivos que incluiu os sorovares: Australis, Bratislava, Autumnalis, Butembo, Castellonis, Bataviae, Canicola, Whitcombi, Cynopteri, Grippotyphosa, Hebdomadis, Copenhageni, Icterohaemorrhagiae, Javanica, Panamá, Pomona, Pyrogenes, Hardjo, Wolffi, Shermani, Tarassovi, Patoc e Sentot.

Os soros foram triados na diluição de 1:100 e os que apresentaram $50 \%$ ou mais de aglutinação foram titulados peloexame de uma série de diluições geométricas de razão dois. O título do soro foi a recíproca da maior diluição que apresentou resultado positivo. Os 
antígenos foram examinados ao microscópio de campo escuro, previamente aos testes, a fim de verificar a mobilidade e a presença de autoaglutinação ou de contaminantes.

\section{Cálculo da soroprevalência}

O provável sorovar infectante foi considerado aquele que apresentou maior título. Na ocorrência de cruzada entre dois ou mais sorovares, o animal foi desconsiderado da análise. Na propriedade, o provável sorovar infectante foi o que apresentou o maior título e o maior número de reações positivas. Uma propriedade foi considerada positiva quando nela foi encontrado pelo menos um animal soropositivo.

Foi calculada a prevalência de propriedades positivas edeanimais soropositivos para qualquer sorovar e para os sorovares individuais, no Estado da Bahia e por estrato amostral.

O delineamento amostral para o cálculo da prevalência de animais soropositivos para a leptospirose bovina no Estado da Bahia empregou uma amostra de grupo estratificada em dois estágios, eem cada estrato amostral, uma amostra de grupo em dois estágios (THRUSFIELD, 1995), onde cada propriedade foi considerada um grupo. Os parâmetros utilizados foram: (a) condição do animal (soropositivo ou soronegativo); (b) região (estrato amostral) a qual pertencia o animal; (c) código do rebanho (para identificar cada grupo); e (d) peso estatístico. O peso estatístico foi calculado conforme (DEAN, 1994) ${ }^{1}$

Para o cálculo da prevalência de propriedades positivas, odelineamento amostral empregou a amos- tra aleatória estratificada (THRUSFIELd, 1995). Os parâmetros necessários foram: (a) condição da propriedade (rebanho reagente ou não reagente); (b) região (estrato amostral) a qual pertencia a propriedade; e(c) peso estatístico. O peso estatístico foi determinado segundo (DEAN, 1994) ${ }^{2}$

\section{RESULTADOS}

Das 10.823 fêmeas bovinas do Estado da Bahia, examinadas no período de março a setembro de 2004, 4.253 foram reagentes na SAM para pelo menos um dos sorovares de Leptospira spp., resultando em uma soroprevalência de 45,42\% (IC 95\% = 42,00\% -48,88\%), com títulos variando de 100 a 12800 . A região 2 foi a que apresentou a maior prevalência de animais reagentes $(48,6 \%$; IC 95\% $=43,68 \%-53,56 \%)$, seguida pelas regiões 1,3 e 4 (Tabela 1).

Osorovar Hardjo (Hardjoprajitno) foi o predominante do total de animais examinados (14,95\%; IC $95 \%=12,59 \%-17,67 \%)$, seguido pelos sorovares Shermani, Wolffi (Tabela 2).

Dentre as 1.414 propriedades investigadas, 1076 (77,93\%; IC 95\% = 75,73\% - 79,99\%) apresentaram pelo menos um animal reagente na SAM para qualquer sorovar (Tabela3). A maior frequência de propriedades positivas ocorreu na região 2 (88,10\%; IC 95\% $=84,16 \%-91,15 \%$ ), seguida das regiões 3, 4 e 1 . O sorovar Hardjo (Hardjoprajitno), com 34,49\% (IC 95\% $=31,93 \%-37,14 \%$ ) de propriedades positivas, foi o sorovar mais prevalente, seguido pelos sorovares Shermani e Wolffi (Tabela 4 e Fig. 2).

Tabela 1 - Soroprevalência para qualquer sorovar de Leptospira spp. em fêmeas bovinas em quatro estratos amostrais no Estado da Bahia, durante o período de março a setembro de 2004.

\begin{tabular}{cccc}
\hline Região & Proporção de reagentes* & Prevalência (\%) & IC 95\% (\%) \\
\hline 1 & $1092 / 3566$ & 42,52 & $35,82-49,51$ \\
2 & $1228 / 2621$ & 48,60 & $43,68-53,56$ \\
3 & $981 / 2142$ & 47,72 & $40,36-55,18$ \\
4 & $952 / 2494$ & 44,18 & $38,13-50,41$ \\
\hline Estado & $4253 / 10823$ & 45,42 & $42,00-48,88$ \\
\hline
\end{tabular}

* = Número de animais reagentes para pelo menos um sorovar de Leptospira spp., por número de animais examinados.

$$
{ }^{1} \text { Peso }=\frac{\text { fêmeas } \geq 24 \text { meses na região }}{\text { fêmeas } \geq 24 \text { meses nas propr. amostradas }} \times \frac{\text { fêmeas } \geq 24 \text { meses na propr. }}{\text { fêmeas } \geq 24 \text { meses amostradas na propr } .}
$$

$$
{ }^{2} \text { Peso }=\frac{n^{o} \text { de propriedades na região }}{n^{o} \text { de propriedades amostradas na região }}
$$




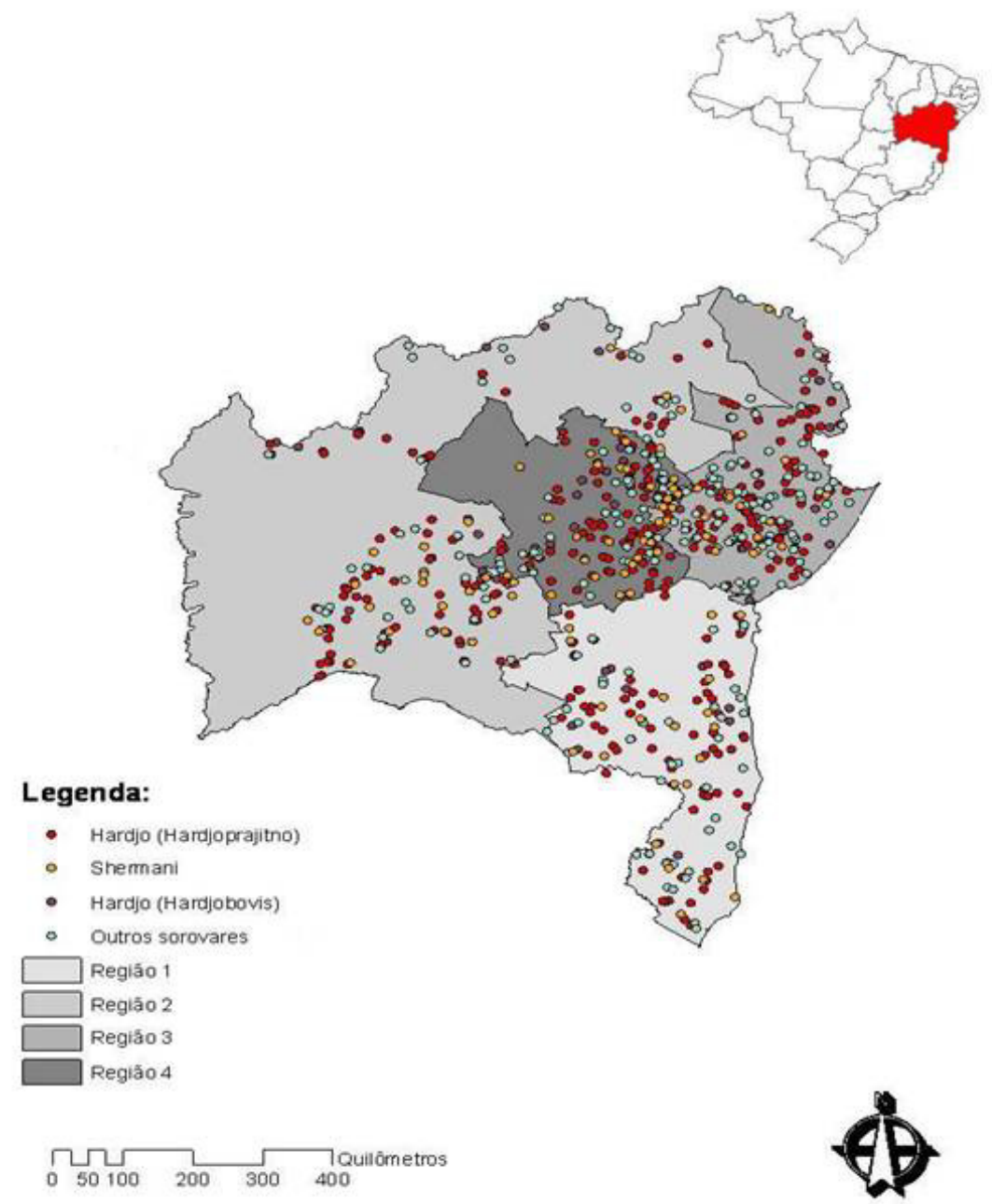

Fig. 2 - Mapa temático do Estado da Bahia, com a distribuição espacial dos sorovares de Leptospira spp., predominantes nas propriedades de acordo com o estrato amostral. Detalhe superior destacando a localização do Estado da Bahia no Brasil.

\section{DISCUSSÃO}

A aplicação da SAM para detecção dos anticorpos anti-Leptospiras spp. em 10.823 soros de fêmeas bovinas em idade reprodutiva, provenientes de 1.414 propriedades permitiu a visualização da abrangência e da distribuição de animais soropositivos para Leptospira spp. noEstado da Bahia eas sorovariedades predominantes. De fato, foram encontrados animais soro reatores para Leptospiras spp. em todas as quatro regiões em queo Estado da Bahia foi subdividido, com uma soroprevalência de $45,42 \%$ em fêmeas bovinas com idade igual ou superior a 24 meses, e em 77,93\% das propriedades examinadas. No Estado de São Paulo, CASTRO et al. (2008) encontraram 49\% de soroprevalência em fêmeas bovinas com idade igual ou superior a 24 meses e $71,3 \%$ das propriedades examinadas com pelo menos um animal soropositivo.
No presente estudo, 23 de 24 sorovares contidos na coleção de antígenos foram detectados nas fêmeas bovinas examinadas e 23 foram evidenciados nas quatroregiões, sendoqueomenornúmerodesorovares detectado nas propriedades pela SAM ocorreu nas regiões 3 e 4 (19 sorovares) e o maior (20 sorovares), nas regiões 1 e 2. Em São Paulo, CASTRO et al. (2008) detectaram 19 de 22 sorovares incluídos na coleção antigênica utilizada no diagnóstico sorológico da leptospirose em fêmeas bovinas e 17 sorovares foram encontrados nas sete regiões em que o Estado de São Paulo foi subdividido.

O sorovar Hardjo (Hardjoprajitno) foi o mais frequente $(14,95 \%)$ no total de animais examinados, seguido pelo Shermani e Wolffi. Similarmente, nas 1.414 propriedades amostradas, os sorovares Hardjo (Hadjoprajitno) (34,49\%) foi o mais frequente, seguido pelos sorovares Shermani e Wolffi, discordando 
dos achados de CALDAS (1991), na Bahia, que encontrou $62,8 \%$ de soropositividade para o sorovar Wolffi e de CASTRO et al. (2008), em São Paulo, que evidenciaram $46 \%$ desoropositividade para o sorovar Hardjo. Flutuações na soropositividadeno decorrer do tempo podem ser atribuídas a diversos fatores, como as espécies animais de contato, os sorovares existentes, as condições ambientais e climáticas, além do manejo e das oportunidades de infecção direta ou indireta (ELLIS, 1984).

CALDASet al.(1979), CALDASetal.(1995/96) e VIEGAS et al.(2001) verificaram predomínio de reatividade sorológica para os sorovares Wolffi, Icterohaemorragiae e Autumnalis, respectivamente, em bovinos no Estado da Bahia. Contudo, nas coleções de antígenos empregadas por eles, não estava incluído o sorovar Hardjo. Inquéritos sorológicos para a leptospirose bovina, realizados em outros estados do Brasil, apontam o sorovar Hardjo (Hardjoprajtino) como o mais frequente em bovinos. De fato, MADrUGA et al. (1980), no Mato Grosso do Sul, MoreIra et al. (1979), AlmeidA et al. (1988) e ARAUJo et al. (2005), em Minas Gerais, Brod et al. (1994) no Rio Grande do Sul, OliveIra et al. (2001) no Estado de Pernambuco, Homem et al. (2001) na Amazônia Oriental, e LiLENBAuM; SouzA (2003) no Rio de Janeiro referiram esta condição.

Tabela 2 - Sorovares de Leptospira spp. prevalentes nas amostras de fêmeas bovinas reagentes em relação ao total de animais positivos do Estado da Bahia, durante o período de março a setembro de 2004.

\begin{tabular}{lccc}
\hline Sorovar & Proporção de reagentes & Prevalência $(\%)$ & IC 95\% $(\%)$ \\
\hline Australis & $58 / 10822$ & 0,88 & {$[0,51-1,1]$} \\
Autumnalis & $150 / 10822$ & 1,54 & {$[1,12-2,10]$} \\
Bataviae & $1 / 10822$ & 0,01 & {$[0,00-0,05]$} \\
Bratislava & $29 / 10822$ & 0,31 & {$[0,11-0,69]$} \\
Butembo & $26 / 10822$ & 0,18 & {$[0,10-0,32]$} \\
Canicola & $1 / 10822$ & 0,01 & {$[0,00-0,04]$} \\
Castellonis & $40 / 10822$ & 0,31 & {$[0,17-0,53]$} \\
Copenhageni & $4 / 10822$ & 0,03 & {$[0,01-0,09]$} \\
Cynopteri & $1 / 10822$ & 0,04 & {$[0,01-0,28]$} \\
Grippotyphosa & $27 / 10822$ & 0,22 & {$[0,12-0,40]$} \\
Hardjo (Hardjoprajitno) & 14,95 & {$[12,59-17,67]$} \\
Hebdomadis & $1384 / 10822$ & 2,07 & {$[0,66-2,74]$} \\
Icterohaemorrhagiae & $227 / 10822$ & $1,53]$ \\
Panamá & $94 / 10822$ & 0,99 & {$[0,01-0,05]$} \\
Patoc & $4 / 10822$ & 0,02 & {$[0,69-1,39]$} \\
Pomona & $169 / 10822$ & 0,98 & {$[0,20-1,45]$} \\
Pyrogenes & $28 / 10822$ & 0,54 & {$[0,51-1,21]$} \\
Sentot & $88 / 10822$ & 0,79 & {$[0,01-0,05]$} \\
Shermani & $5 / 10822$ & 0,02 & {$[4,05-6,01]$} \\
Tarassovi & $459 / 10822$ & 4,94 & {$[0,46-1,05]$} \\
Whitcombi & $76 / 10822$ & 0,69 & {$[0,03-0,19]$} \\
Wolffi & $11 / 10822$ & 0,08 & {$[2,92-4,36]$} \\
\hline
\end{tabular}

Tabela 3 - Frequência de propriedades com pelo menos um animal soropositivo para Leptospira spp. nos quatro estratos amostrais no Estado da Bahia, durante o período de março a setembro de 2004.

\begin{tabular}{cccc}
\hline Região & Proporção de propriedades positivas* & Prevalência (\%) & IC 95\% (\%) \\
\hline 1 & $239 / 383$ & 62,40 & {$[57,43-67,13]$} \\
2 & $296 / 336$ & 88,10 & {$[84,16-91,15]$} \\
3 & $282 / 364$ & 77,47 & {$[72,88-81,49]$} \\
4 & $259 / 331$ & 78,25 & {$[73,47-82,37]$} \\
\hline Estado & $1076 / 1414$ & 77,93 & {$[75,73-79,99]$} \\
\hline
\end{tabular}

* = Número de propriedades com pelo menos um animal soropositivo para Leptospira spp., por número de propriedades amostradas. 
Tabela 4 - Sorovares de Leptospira spp. prevalentes nas propriedades reagentes em relação ao total de propriedades positivas do Estado da Bahia, durante o período de março a setembro de 2004.

\begin{tabular}{lrrr}
\hline Sorovar & Proporção de propriedades positivas & Prevalência (\%) & IC 95\% (\%) \\
\hline Australis & $10 / 1414$ & 0,72 & {$[0,37-1,37]$} \\
Autumnalis & $25 / 1414$ & 1,77 & {$[1,18-2,65]$} \\
Bratislava & $7 / 1414$ & 0,49 & {$[0,22-1,08]$} \\
Butembo & $4 / 1414$ & 0,25 & {$[0,09-0,68]$} \\
Castellonis & $5 / 1414$ & 0,44 & {$[0,18-1,07]$} \\
Grippotyphosa & $7 / 1414$ & 0,60 & {$[0,28-1,28]$} \\
Hardjo (Hardjoprajitno) & $485 / 1414$ & 34,49 & {$[31,93-37,14]$} \\
Hebdomadis & $53 / 1414$ & 3,06 & {$[2,93-5,07]$} \\
Icterohaemorrhagiae & $12 / 1414$ & 0,85 & {$[0,47-1,54]$} \\
Patoc & $40 / 1414$ & 3,15 & {$[2,29-4,33]$} \\
Pomona & $8 / 1414$ & 0,61 & {$[0,30-1,27]$} \\
Pyrogenes & $19 / 1414$ & 1,31 & {$[0,82-2,08]$} \\
Sentot & $2 / 1414$ & 0,21 & {$[0,05-0,84]$} \\
Shermani & $118 / 1414$ & 8,17 & {$[6,79-9,79]$} \\
Tarassovi & $13 / 1414$ & 1,12 & {$[0,64-1,94]$} \\
Whitcombi & $1 / 1414$ & 0,11 & {$[0,01-0,74]$} \\
Wolffi & $76 / 1414$ & 5,34 & {$[4,25-6,70]$} \\
\hline
\end{tabular}

Além da presença do sorovar Hardjo (Hardjoprajitno), cuja transmissão usualmente ocorre entre bovinos, em alguns rebanhos ou regiões poderiam estar ocorrendo infecções acidentais por outros sorovares, cuja transmissão indireta está associada ao contato com o meioambientecontaminadopor leptospiras oriundas de espécies silvestres ou de outras espécies domésticas. Cervídeos, capivaras e outras espécies silvestres podem atuar como reservatórios de Leptospiras spp. para os rebanhos ao encontrar ohabitat satisfatório(CASTROetal.,2008).Sorovares acidentais como Hebdomadis e Pyrogenes, detectados nesse estudo e cujas descrições são relacionadas com animais silvestres (SANTA RosA et al., 1975; SANTA RosAetal.,1980), levantam a suspeita doenvolvimento dessas espécies da fauna como reservatórios destes sorovares para os bovinos.

Osorovar Shermani, que aparece como o segundo mais freqüente, reforça a importância da ampla composição da coleção de antígenos. Este sorovar foi isolado pela primeira vez de um roedor (Proechimys semispinosus) no Panamá em 1982 (Sulzer et al., 1982). No Brasil, há relato de isolamento deste sorovar de roedores no Mato Grosso(Lins; SANTA RosA, 1976). Em Rondônia, AGUIAR (2006) evidenciounaSAMosorovar Shermani como o terceiro mais frequente, precedido pelos sorovares Hardjo e Wolffi.

Em resumo, o presente trabalho levanta a hipótese de disseminação de Leptospira spp. por todo o Estado da Bahia, com o sorovar Hardjo (Hardjoprajitno) sendo o mais prevalente em fêmeas bovinas em idade reprodutiva, reforçando os achados clássicos de que esse sorovaré o mais comumente envolvido nas infecções por Leptospira spp. nos rebanhos bovinos brasileiros à semelhança do que ocorre mundialmente.

\section{AGRADECIMENTOS}

Agradecemos ao Ministério da Agricultura, Pecuária e Abastecimento e à Agência de Defesa Agropecuária do Estado da Bahia pela colheita, georreferenciamento e autorização para a utilização dos soros de bovinos destinados ao PNCBET.

\section{REFERENNCIAS}

AGUIAR, D.M.; GENNARI, S.M.; CAVALCANTE, G.T.; LABRUNA, M.B.; VASCONCELLOS, S.A.; RODRIGUES, A.A.R.; MORAIS, Z.M.; CAMARGO, L.M.A.

Seroprevalence of Leptospira spp. in cattle from Monte Negro municipality, western Amazon. Pesquisa Veterinaria Brasileira, v.26, n.2, p.102-104, 2006.

ALMEIDA, S.C.A.; SILVA, P.L.; BARBOSA, F.C.; GOUVEIA, M.A.V.; OLIVEIRA, P.R.; MANEDE, D.O. Levantamento sorológico em dois surtos de leptospirose bovina, em Uberlândia, Triangulo Mineiro. Arquivos Brasileiros de Medicina Veterinária e Zootecnia, v.40, n.6, p.415-423, 1988.

ALVES, A.J.S. Caracterização da Brucelose Bovina no Estado da Bahia. 2007. 87p. Dissertação (Mestrado em Medicina Veterinária) - Faculdade de Medicina Veterinária e Zootecnia, Universidade de São Paulo, São Paulo, 2007. 
ARAUJO, V E.M.; MOREIRA, E.C.; NAVEDA, L.A.B.; SILVA, J.A.; CONTRERAS, R.L. Frequência de aglutininas anti-Leptospira interrogans em soros sanguíneos de bovinos em Minas Gerais, de 1980 a 2002. Arquivos Brasileiros de Medicina Veterinária e Zootecnia, v.57, n.4, p.430-435, 2005.

BROD, C.S.; MARTINS, L.F.S.; NUSSBAUN, J.R.; FEHLBERG, M.F.B.; FURTADO, L.R.I.; ROSADO, R.L.I. Leptospirose bovina na região sul do Estado do Rio Grande do Sul. A Hora Veterinária, v.14, p.1520, 1994 .

CALDAS, E M.; SAMPAIO, M.B.; COSTA, E.; MIRANDA, G. Estudo epidemiológico de surto de leptospirose ocorrido na cidade do Salvador, Bahia, em maio e junho de 1978. Revista do Instituto Adolfo Lutz, v.39, n.1, p.85-94, 1979.

CALDAS, E.M. Comportamento de estirpes apatogênicas no diagnóstico sorológico de leptospirose em animais. Arquivos da Escola de Medicina Veterinária da Universidade Federal da Bahia, v.14, n.1, p.324, 1991

CALDAS, E.M.; VIEGAS, E.A.; VIEGAS, S.A.R.A.; REIS, R.S. Aglutininas anti-leptospira em hemosoro de animais domésticos no estado da bahia. Arquivos da Escola de Medicina Veterinária da Universidade Federal da Bahia, v.18, n.1, p.268-280, 1995/1996.

CASTRO, V.; AZEVEDO, S.S.; GOTTI, T.B.; BATISTA, C.S.A.; GENTILI, J.; MORAIS, Z.M.; SOUZA, G.O.; VASCONCELLOS, S.A.; GENOVEZ, M.E.

Soroprevalência da leptospirose em fêmeas bovinas em idade reprodutiva no Estado de São Paulo, Brasil. Arquivos do Instituto Biológico, São Paulo, v.75, n.1, p.3$11,2008$.

COLE J.R.; SULZER, C.R.; PURSELL, A.R. Improved microtechnique for the leptospiral microscopic agglutination test. Applied Microbiology, v.25, n.6, p.976980, 1973.

DEAN, A.G. EpiInfo version 6: a word-processing, database, and statistic program for public health on IBM-compatible microcomputers. Atlanta: Center for Diseases Control and Prevention, 1994. 601p.

ELLIS, W.A. Bovine leptospirosis in the tropics: prevalence, pathogenesis and control. Preventive Veterinary Medicine, v.2, p.411-421, 1984.

ELLIS, W.A.; O'BRIEN, J.J.; CASSELLS, J.A.; NEILL, S.D.; HANNA, J. Excretion of Leptospira Interrogans serovar hardjo following calving or abortion. Research veterinary science, v.39, p.296-298, 1985.

ELLIS, W.A. Leptospirosis as a cause of reproductive failure. Veterinary Clinics of North America: Food Animal Practice, v.10, p.463-478, 1994.
FAINE, S.; ADLER, B.; BOLIN, C.; PEROLAT, P. Leptospira and Leptospirosis. 2nd ed. Melbourne: MedSci, 1999. 272p.

FUNDAÇÃO NACIONAL DE SAÚDE (Brasil). Centro Nacional de Epidemiologia. Coordenadoria de Controle de Zoonoses e Animais Peçonhentos. Manual de leptospirose. Brasília, 1995. 98p.

GALTON, M.M.; SULZER, C.R.; SANTA ROSA, C.A.; FÍELDS, M.J. Application of a microtechnique to the agglutination test for leptospiral antibodies. Applied Microbiology, v.13, n.l, p.81-85, 1965.

GROOMS, D.L. Reproductive losses caused by bovine viral diarrhea virus and leptospirosis. Theriogenology, v.66, p.624-628, 2006.

HOMEM, V.S.F.; HEINEMANN, M.B.; MORAES, Z.M.; VASCONCELLOS, S.A.; FERREIRA, F.; FERREIRA NETO, J.S. Estudo epidemiológico da leptospirose bovina e humana na Amazônia oriental brasileira. Revista da Sociedade Brasileira de Medicina Tropical, v.34, n.2, p.173-180, 2001.

IBGE - Instituto Brasileiro de Geografia e Estatística. Sistema IBGE de Recuperação Automática - SIDRA. Pesquisa Pecuária Municipal, 2007. Disponível em: <http://www.sidra.ibge.gov.br/bda/tabela/ listabl.asp? $\mathrm{c}=73 \& \mathrm{z}=\mathrm{t} \& \mathrm{o}=20>$. Acesso em: 5 out. 2009.

LILENBAUM, W.; SOUZA, G.N. Factors associated with bovine leptospirosis in Rio de Janeiro, Brazil. Research in Veterinary Science, v.75, p.249-251, 2003.

LINS, Z.C.; SANTA ROSA, C.A. Investigações epidemiológicas preliminaries sobre leptospiroses em Humboldt, Aripuanã, Mato Grosso. Acta Amazônica, v.6, n.4, p.46-53, 1976.

MADRUGA, C.R.; AYCARDI, E.; PUTT, N.

Frequencia de aglutininas anti-leptospira em bovinos de corte da região sul de cerrado do Estado do Mato Grosso. Arquivos da Escola de Veterinária da Universidade Federal de Minas Gerais, v. 2, n.2, p.245$249,1980$.

MOREIRA, E.C.; SILVA, J.A; VIANA, F.C.; SANTOS, W.L.M.; ANSELMO, F.P.; LEITE, R.C. Leptospirose bovina I: Aglutininas anti-leptospira em soros sanguíneos de bovinos de Minas Gerais. Arquivos da Escola de Veterinária da Universidade Federal de Minas Gerais, v.31, n.3, p.375-388, 1979.

OLIVEIRA, A.A.; MOTA, R.A.; PEREIRA, G.C.; LANGONI, H.; SOUZA, M.I.; NAVEGANTES, W.A.; SA, M.E. Seroprevalence of bovine leptospirosis in Garanhuns municipal district, Pernambuco State, Brazil. Onderstepoort Journal of Veterinary Research., v.68, n.4, p.275-279, 2001. 
SANTA ROSA, C.A.; SULZER, C.R.; GIORGI, W.; SILVA, A.S. da; YANAGUITA, R.M.; LOBÃO, A.O. Leptospirosis in wildlife in Brazil: isolation of a new serotype in pyrogenes group. American Journal of Veterinary Research, v.36, p.1363-1365, 1975.

SANTA ROSA, C.A.; SULZER, C.R.; YANAGUITA, R.M.; SILVA, A.S. da Leptospirosis in wildlife ibn Brazil: isolation of serovars Canicola, Pyrogenes and Grippotyphosa. International Journal of Zoonosis, v.7, p.40-43, 1980.

SULZER, K.; POPE, V.; ROGERS, F. New leptospiral serotypes (serovars) from the Western Hemisphere isolated during 1964 through 1970. Revista Latinoamericana de Microbiologia, v.24, p.15-17, 1982.

THRUSFIELD, M. Veterinary epidemiology. 2 ed. Cambridge: Blackwell Science, 1995. 479p.

VIEGAS, S.A.R.A.; CALDAS, E.M.; OLIVEIRA, E.M.D. Aglutininas anti-leptospira em hemosoro de animais domésticos de diferentes espécies, no Estado da Bahia, 1997/1999. Revista Brasileira de Saúde e Produção Animal, v.1, p.1-6, 2001.

Recebido em 19/4/09

Aceito em 6/10/09 